\title{
Importancia y aporte del sector cooperativo en el desarrollo humano: un análisis empírico para el caso costarricense
}

\author{
Federico Li-Bonilla ${ }^{\star}$, José F. Sandoval ${ }^{\star *}$
}

* Doctor en Ciencias de la Administración. Coordinador de la Carrera de Administración de Empresas con énfasis en cooperativas y asociativas, Universidad Estatal a Distancia, Costa Rica.

Correo electrónico: fli@uned.ac.cr

** Magíster en Política Económica con especialidad en Economía Ecológica y Desarrollo Sostenible. Magíster en Derechos Humanos. Docente de la cátedra de Economía, Universidad Estatal a Distancia, Costa Rica.

Correo electrónico:

jsandoval@uned.ac.cr

Recibido: 2 de abril del 2013

Aprobado: 4 de septiembre del 2013

Cómo citar este artículo: Li-Bonilla, F. y Sandoval, J.F. (2013). Importancia y aporte del sector cooperativo en el desarrollo humano: un análisis empírico para el caso costarricense. Cooperativismo \& Desarrollo, 21(103), 9-17.

\section{Resumen}

Para la Organización de las Naciones Unidas (ONU), el modelo cooperativo es un pilar de suma importancia en el desarrollo de muchas comunidades, países y regiones a nivel global. Aunque es común inferir la existencia de una relación directa entre el cooperativismo y el desarrollo socioeconómico de las regiones, esta relación ha sido escasamente estudiada desde el punto de vista económico. Es por ello que en este artículo se presentan los principales hallazgos surgidos a partir del uso de la teoría económica y de técnicas econométricas para medir esa relación. El análisis empírico se realiza con base en una muestra de datos de corte transversal para los 81 cantones del país, y busca abrir una nueva línea de investigación que permita medir la contribución del sector cooperativo al desarrollo costarricense y construir un nuevo pensamiento sobre el desarrollo del sector cooperativo en Costa Rica.

Palabras clave: cooperativismo, desarrollo humano, desarrollo territorial, municipalidades.

\section{The Cooperative Sector's Significance and Contribution to Human Development: an Empirical Analysis of Costa Rica}

\begin{abstract}
According to the United Nations, the cooperative model is a vitally important pilar of development in many communities, countries and regions around the world. Although it is common to infer that there is a direct relationship between cooperativism and socioeconomic development in the regions, there are very few studies that look at this from an economic point of view. This article therefore presents the main findings that emerged from the use of economic theory and econometric techniques to measure the relationship. The empirical analysis is conducted based on a cross-section of data for the country's 81 cantons, and seeks to open a new line of research that will allow the cooperative sector's contribution to Costa Rican development to be measured, and build new thinking about the development of this sector in Costa Rica.
\end{abstract}

Keywords: cooperativism, human development, territorial development, municipalities.

\section{Importância e contribuição do setor cooperativo no desenvolvimento humano: uma análise empírica para o caso costa-riquenho}

\section{Resumo}

Para a Organização das Nações Unidas (oNU), o modelo cooperativo é um pilar de suma importância no desenvolvimento de muitas comunidades, países e regiões em nível global. Embora seja comum inferir a existência de uma relação direta entre o cooperativismo e o desenvolvimento socioeconômico das regiões, essa relação tem sido escassamente estudada desde o ponto de vista econômico. Nesse sentido, neste artigo, apresentam-se as principais descobertas surgidas a partir do uso da teoria econômica e de técnicas econométricas para medir essa relação. A análise empírica se realiza com base em uma amostra de dados de corte transversal para os 81 cantões do país e procura abrir uma nova linha de pesquisa que permita medir a contribuição do setor cooperativo para o desenvolvimento costa-riquenho e construir um novo pensamento sobre o desenvolvimento do setor cooperativo na Costa Rica.

Palavras-chave: cooperativismo, desenvolvimento humano, desenvolvimento territorial, municipalidades. 


\section{Las cooperativas y el desarrollo regional}

El cooperativismo se ha erigido en modelo de desarrollo mundial, con una gran diversidad de formas de realizar negocios según sea la necesidad en el continente, las subregiones en que se sitúe y la comprensión del modelo cooperativo que se tenga según sea su nivel de desarrollo e idiosincrasia de la región.

Es así como las cooperativas contribuyen a la creación de empleo digno y productivo. Según la Asociación Cooperativa Internacional (ACI) (2012), las cooperativas son las responsables de crear, entre familiares y su comunidad, no menos de 100 millones de puestos laborales. Así por ejemplo, tenemos que en Alemania las 8.100 empresas cooperativas generan 440.000 puestos laborales; en Canadá dan trabajo a 160.000 personas, en tanto que en Francia se emplean más de 700.000 personas. En África, tan solo en Kenia, las organizaciones cooperativas son responsables de darles empleo a 250.000 seres humanos, mientras que en Suramérica se han creado más de 488.000 puestos laborales por esa vía. Aunado a esto, el movimiento cooperativo es responsable de la existencia de trabajos indirectos e inducidos. Se entiende por empleo indirecto el generado por los encadenamientos hacia delante y atrás, que las cooperativas establecen con otras empresas similares o con empresas no cooperativas. En cuanto al empleo inducido, es el que se genera con los salarios que gastan los trabajadores y miembros de las cooperativas en su medio económico.

En investigaciones realizadas se ha llegado a la conclusión de que por cada cien puestos laborales, el cooperativismo es responsable de la creación de setenta empleos indirectos, siendo una relación de 10 a 7 .

Las empresas cooperativas fomentan la autoayuda en comunidades en las que las inversiones estatales y las privadas son muy limitadas. Esto permite, por un lado, satisfacer las necesidades en dichas comunidades $y$, por otro, mejorar el nivel de vida de las personas que interaccionan con la cooperativa.

Otro ejemplo de desarrollo regional es la experiencia de la India con el cooperativismo agrario, el cual ha concebido la revolución verde y la blanca; esta última se refiere al desarrollo lechero, el cual se ha constituido en generador de puestos de trabajo directo, indirecto $\mathrm{e}$ inducido en la gran zona rural, en donde existen más de 150.000 organizaciones cooperativas, que han generado millones de empleos. En cuanto a la población que cobija, es preciso señalar que el modelo cooperativo de la India brinda soluciones financieras, de comercialización, insumos y distribución a más de 157 millones de productores agrícolas y rurales. Siendo que en este subcontinente las empresas cooperativas vinculadas con la actividad lechera dan trabajo aproximadamente a 12,4 millones de familias agrícolas, queda demostrada la eficacia del cooperativismo como medio para generar empleo y mejorar los medios de vida de los pobres.

\section{El cooperativismo en Costa Rica}

El cooperativismo en nuestro país empieza de una manera incipiente; sin embargo, ya en 1893 se dan los primeros emprendimientos cooperativos (Aguilar y Fallas, 1990). Posteriormente sobreviene la Gran Depresión, entre 1923 y 1943, perIodo durante el cual se crean una gran cantidad de cooperativas. En Costa Rica, el cooperativismo formal y propiamente dicho se inició en los años veinte del siglo pasado. Al respecto, conviene señalar que desde 1919 el entonces presidente de la República, don Julio Acosta García, al restablecer la Constitución de 1897, incluyó el artículo 58, el cual dice: "El Estado fomentará las formas de actividades cooperativas como medio de facilitar mejores condiciones de vida a los trabajadores" (Li, 1989, p. 5).

La Ley 861 del 6 de enero de 1947 creó la sección de Fomento de Cooperativas Agrícolas e Industriales en el Banco Nacional de Costa Rica, primer organismo técnico nacional con la capacidad para ayudar a este tipo de cooperativas. Según Li (1989), esta sección impulsó varias cooperativas, de las cuales actualmente subsisten la Cooperativa Agroindustrial La Victoria, RL (1943) y la cooperativa Agroindustrial y Servicios Múltiples de Productores de Leche, RL (1947).

El cooperativismo en Costa Rica ha tenido la venia de los políticos de turno en diferentes épocas, tanto en la Primera República como en la Segunda República. Así, la primera legislación en materia cooperativa data del 26 de agosto de 1943, cuando se emitió el Código de Trabajo con su capítulo III dedicado a las asociaciones cooperativas.

La Asamblea Legislativa (1982), por su parte, define el concepto de cooperativa en la Ley 6756 de Asociaciones Cooperativas y Creación del Instituto de Fomento Cooperativo (Infocoop) de 1982, cuyo artículo 2 plantea lo siguiente:

las asociaciones cooperativas son grupos de personas unidos de forma voluntaria y democrática, en donde el capital no es la parte importante del cooperativismo. La 
constitución de una cooperativa obedece el satisfacer el mejoramiento social y económico de tal forma que sea individual y grupal. Para lo cual existen diferentes tipos de gestión cooperativa y de actividades económicas.

Como se aprecia, en Costa Rica el cooperativismo no sólo está contemplado en la Constitución, sino que está amparado por una ley específica. De ahí que el fundamento del desarrollo cooperativo nacional se haya convertido en un modelo óptimo de desarrollo nacional, que no solamente reparte equidad social, sino también bienestar económico a la población costarricense.

\section{Tipos de cooperativas}

El tipo de cooperativa en cada región y país varía según sus necesidades e idiosincrasia; es así como tenemos cooperativas de corte financiero como los bancos, las cajas y las cooperativas de ahorro y crédito. También hay empresas cooperativas de servicios, entre ellas, las empresas de transporte, que brindan servicio público con líneas de buses o taxis; además, existen las cooperativas de producción, principalmente aquellas que se dedican a la industrialización de cárnicos, leche y sus derivados, azúcar, café, sal, camarones, entre otros.

En Costa Rica, la Ley 6756 de Asociaciones Cooperativas y Creación del Instituto de Fomento Cooperativo (Infocoop) de 1982, define doce tipos diferenciados, de acuerdo con la manera como las cooperativas de realizar operaciones. Pero, además, las cooperativas se dividen en tres grandes grupos según sea la injerencia de la participación del asociado en ella. Se cuenta con las de gestión o tradicionales, en las cuales la tónica es que los asociados participan con un capital social y no es necesario que sean trabajadores directos; además, los trabajadores pueden ser simplemente asalariados de la cooperativa. En la cooperativa de autogestión, lo fundamental es que todos sus trabajadores deben ser asociados, con las excepciones que dicta la ley para este caso. Por último están las cooperativas de cogestión, en las que generalmente los cooperativistas se asocian con el Estado o alguna empresa y conforman una cooperativa.

\section{Estudio econométrico}

En este apartado se presentan los resultados de una serie de pruebas econométricas realizadas para medir la relación existente entre la afiliación en cooperativas y el nivel de desarrollo humano cantonal, el cual es enfocado de acuerdo con diversos indicadores de desarrollo que estiman algunas instituciones del país y externas. Entre otros, se utilizan los siguientes indicadores: Índice de Desarrollo Humano (IDH), Índice de Desarrollo Social (IDS), índice de cobertura de educación secundaria, extensión territorial, urbanismo y educación.

Las ecuaciones utilizadas en el estudio se establecieron básicamente por características exógenas a las poblaciones y la tenencia de ciertas formas de capital humano, específicamente el concepto educación, ingreso y nivel de urbanismo de la localidad. Las pruebas econométricas se realizaron empleando las herramientas estadísticas de análisis de datos de la hoja de cálculo Microsoft Excel.

Para el análisis se dispuso de una muestra de datos de corte transversal para los 81 cantones que integran el país. El conjunto de datos utilizado en el análisis empírico provino de encuestas y censos del Instituto Nacional de Estadísticas y Censos, indicadores del Ministerio de Planificación Nacional y Política Económica y del Programa de las Naciones Unidas para el Desarrollo (PNUD) $(2007,2010)$.

Los datos utilizados a nivel cantonal son los siguientes: Índice de Desarrollo Humano, Índice de Desarrollo Social, índice de cobertura de educación secundaria, extensión territorial, población, porcentaje de urbanismo, consumo de energía eléctrica, cantidad de cooperativas por cantón y población perteneciente a las cooperativas. En general, con la finalidad de contar con una base de datos lo más homogénea posible, las variables tienen como base el 2005.

Para este caso específico, el estudio se enfocó en la búsqueda de evidencia empírica sobre la importancia de las cooperativas en el desarrollo humano del país, por lo que se planteó una relación entre indicadores de desarrollo y las variables regionales. El enfoque adoptado sigue básicamente la metodología de Barro (1991) y pretende describir el conjunto de determinantes de la tasa de desarrollo socioeconómico.

\section{Ecuación estimada}

La relación general entre el indicador de desarrollo (ID) y las variables independientes, puede expresarse de la siguiente forma: IDi = $\mathrm{f}(\mathrm{Ci} Y \mathrm{Y}, \mathrm{EDi}, \mathrm{Ri})(1)$, donde IDi corresponde al indicador de desarrollo de cada cantón, $\mathrm{Ci}$ al indicador de disponibilidad de cooperativas, $\mathrm{Yi}$ al nivel de ingreso, EDi a las variables de capital humano (educación) y $\mathrm{Ri}$ a las variables regionales (urbanismo, área). 
La relación funcional entre la variable dependiente y cada variable independiente dependerá del tipo de dato que se utilice para representarla. Sin embargo, para el caso de ingreso, disponibilidad de cooperativas y capital humano, la hipótesis que se someterá a prueba es que poseen una relación directa; es decir, a mayor nivel de ingreso, disponibilidad de cooperativas y de capital humano, se tendrá mayor nivel de desarrollo (un valor más alto del índice de desarrollo utilizado).

Maddala (1996) indica que antes de realizar el análisis estadístico con los datos económicos, es necesaria una formulación matemática clara de la teoría económica pertinente. Sin embargo, la teoría económica rara vez informa sobre estas formas funcionales, por lo cual es preciso utilizar métodos econométricos para elegir la forma apropiada.

Con el anterior propósito, se consideraron tres tipos de especificaciones funcionales para estimar la ecuación (1); estas son, la forma funcional lineal, la forma funcional semilogarítmica y la forma logarítmica. A su vez, la especificación semilogarítmica se puede representar mediante dos formas diferentes: la forma lineal-logarítmica (LINLOG) y la formulación logarítmica-lineal (LOGLIN).

En detalle, las formas funcionales específicas estimadas son las siguientes:

a) Forma lineal (linlin), en donde IDi $=\alpha_{0}+\alpha_{1} \times C_{i}$ $+\alpha_{2} \times Y_{i}+\alpha_{3} \times E_{i}+\alpha_{4} \times R_{i}+\mu_{i}(2)$

Esta formulación proporciona parámetros que muestran cómo se modifica la variable explicada ante cambios unitarios en las variables explicativas.

b) Forma lineal logarítmica (LINLOG), en donde $\mathrm{e}^{\mathrm{IDi}}=$ $\alpha_{0} C_{i}^{a 1} \times Y_{i}^{a 2} \times E_{i}^{a 3} \times R_{i}^{a 4} \times e^{\mu i}$, que se puede expresar de forma alternativa como:

$\mathrm{IDi}=\alpha_{0}^{\prime}+\alpha_{1}^{\prime} \times \ln \left(\mathrm{C}_{\mathrm{i}}\right)+\alpha_{2}^{\prime} \times \ln \left(\mathrm{Y}_{\mathrm{i}}\right)+\alpha_{3}^{\prime} \times \ln \left(\mathrm{E}_{\mathrm{i}}\right)$ $+\alpha^{\prime} \times \ln \left(\mathrm{R}_{\mathrm{i}}\right)+\mu_{\mathrm{i}}^{\prime}(3)$, donde ln es el logaritmo natural (base e).

Esta forma funcional proporciona las elasticidades de la variable explicada con respecto a las variables explicativas. En este caso los coeficientes de elasticidad afectan a la variable por medio del logaritmo de las variables explicativas, de forma tal que la variable independiente varía a una tasa menor ante variaciones en las variables independientes. c) Forma funcional logarítmica lineal (LOGLIN), en donde

$\mathrm{IDi}=\mathrm{e}^{(\alpha 0+\alpha 1 \times \mathrm{Ci}+\alpha 2 \times \mathrm{Yi}+\alpha 3 \times \mathrm{Ei}+\alpha 4 \times \mathrm{Ri}+\mu \mathrm{i})}$, que se puede expresar de forma alternativa como:

$\ln \left(\mathrm{ID}_{\mathrm{i}}\right)=\alpha_{0}^{\prime}+\alpha_{1}^{\prime} \times \mathrm{C}_{\mathrm{i}}+\alpha_{2}^{\prime} \times \mathrm{Y}_{\mathrm{i}}+\alpha_{3}^{\prime} \times \mathrm{E}_{\mathrm{i}}+\alpha_{4}^{\prime} \times$ $\mathrm{R}_{\mathrm{i}}+\mu_{\mathrm{i}}^{\prime}(4)$

Esta forma funcional permite derivar elasticidades no constantes. El valor de cada coeficiente de elasticidad no es independiente del valor de la variable asociada con dicho coeficiente. Esto indica que conforme se aumenta el valor de alguna de las variables explicativas, el impacto que esta tiene sobre el Índice de Desarrollo Humano es cada vez mayor.

d) Forma funcional logarítmica (LOGLOG), en donde IDi $=\alpha_{0} C_{i}^{\alpha 1} \times Y_{i}^{\alpha 2} \times E_{i}^{\alpha 3} \times R_{i}^{\alpha 4} \times e^{\mu i}$, que se puede expresar de forma alternativa como:

$\ln \left(\mathrm{ID}_{\mathrm{i}}\right)=\alpha_{0}^{\prime}+\alpha_{1}^{\prime} \times \ln \left(\mathrm{C}_{\mathrm{i}}\right)+\alpha_{2}^{\prime} \times \ln \left(\mathrm{Y}_{\mathrm{i}}\right)+\alpha_{3}^{\prime} \times$ $\ln \left(\mathrm{E}_{\mathrm{i}}\right)+\alpha_{4}^{\prime} \times \ln \left(\mathrm{R}_{\mathrm{i}}\right)+\mu_{\mathrm{i}}^{\prime}(5)$.

Mediante esta forma funcional se obtienen elasticidades constantes (a’i), de forma que una alta elasticidad indicaría que un cambio en la variable explicativa tiene un gran impacto sobre el Índice de Desarrollo; lo contrario ocurriría en caso de una baja elasticidad.

\section{Estrategia de estimación}

La disponibilidad de más de dos indicadores para estimar la variable explicada "desarrollo socioeconómico" (ingreso, índice de desarrollo social e índice de desarrollo humano), más una cantidad grande de datos para representar las variables explicativas, proporcionaron distintos conjuntos de variables para estimar la ecuación básica del modelo.

En esta etapa de ajuste, para discriminar entre los diferentes conjuntos de datos y elegir aquellos que mejor representaran la relación que se estudia, la estrategia seguida fue estimar la forma lineal (LINLIN), utilizando como variables explicadas, los diferentes índices de desarrollo cantonales. El mejor resultado se obtuvo con el uso del Índice de Desarrollo Humano (IDH) cantonal de la Naciones Unidas, como variable explicada, y como variables explicativas las siguientes: proporción de población afiliada a cooperativas en relación con la población total de cada cantón (cooperativismo); el consumo de energía eléctrica (como proxy al nivel de 
ingreso), la proporción de personas con secundaria en relación con la población total (educación), el porcentaje de urbanismo (urbanismo) y el área en $\mathrm{km}^{2}$ por cantón (área).

El mejor ajuste lineal obtenido (tabla 1) relaciona el IDH con la proporción de la población cantonal en cooperativas, el nivel de ingreso cantonal, nivel de educación, nivel de urbanismo y el área en $\mathrm{km}^{2}$ (estas dos últimas como variables regionales).

Como se aprecia en la tabla 1 , los coeficientes estimados confirman las hipótesis de análisis, dado que se espera que el nivel de cooperativismo, ingreso, educación y urbanismo muestren una relación positiva con respecto al nivel de desarrollo de cada cantón, mientras que la extensión o área del cantón presentará una relación inversa.

Debido a los resultados satisfactorios obtenidos con las definiciones de las variables ya mencionadas, se efectuaron regresiones con las formas funcionales propuestas con el fin de determinar el modelo apropiado para representar la relación entre la variable dependiente y las variables independientes. La tabla 2 presenta los resultados de la evaluación de estas formas funcionales.

Como puede observarse a partir de la tabla 2, en general los modelos estimados representaron adecuadamente la relación entre el nivel de desarrollo, según el IDH cantonal y las variables explicativas (cooperativismo, ingreso, educación, urbanismo y área). En todos los casos el estadístico F es altamente significativo, los coeficientes de correlación superiores a 0,90 y más del $80 \%$ de la variabilidad del IDH, según cada modelo, es explicada por las regresiones.

En virtud de los resultados obtenidos, se optó por las formas funcionales LINLIN (ecuación 2) y LINLOG (ecuación 3) para modelizar la relación entre las variables.

Tabla 1. Resultado de la estimación del modelo lineal: LINLIN

\begin{tabular}{|l|c|c|c|c|}
\hline \multicolumn{1}{|c|}{ Variable } & Coeficientes estimados & $\begin{array}{c}\text { Error } \\
\text { estándar }\end{array}$ & Estadístico t & Valor-p \\
\hline Constante & 0,55076603 & 0,01652553 & 33,32818522 & 0,00000000 \\
\hline Cooperativismo & 0,00058376 & 0,00451338 & 0,12933981 & 0,89743502 \\
\hline Ingreso & 0,00007660 & 0,00000621 & 12,34079974 & 0,00000000 \\
\hline Educación & 0,04401921 & 0,15936415 & 0,27621777 & 0,78314053 \\
\hline Urbanismo & 0,01628975 & 0,01656188 & 0,98356919 & 0,32848974 \\
\hline Área & $-0,00001949$ & 0,00000460 & $-4,23435808$ & 0,00006429 \\
\hline
\end{tabular}

Estadísticas de la regresión

Coeficiente de correlación múltiple:

Coeficiente de determinación $\mathrm{R}^{2}$ :

$\mathrm{R}^{2}$ ajustado:

Error típico:

Observaciones:
0,92420466

0,85415425

0,84443120

0,02692672

81

Fuente: elaboración propia

Tabla 2. Resultado de la estimación de diferentes formas funcionales

\begin{tabular}{|l|c|c|c|c|c|c|}
\hline \multicolumn{1}{|c|}{$\begin{array}{c}\text { Forma } \\
\text { funcional }\end{array}$} & $\begin{array}{c}\text { Coeficiente } \\
\text { de correlación } \\
\text { múltiple }\end{array}$ & $\begin{array}{c}\text { Coeficiente de } \\
\text { determinación } \\
\mathbf{R}^{2}\end{array}$ & $\mathbf{R}^{2}$ ajustado & Error típico & F & $\begin{array}{c}\text { Valor crítico } \\
\text { de } F\end{array}$ \\
\hline Lin-Lin & 0,92420466 & 0,85415425 & 0,84443120 & 0,02692672 & 87,848 & 0,00000000 \\
\hline Lin-Log & 0,92116067 & 0,84853698 & 0,83843944 & 0,02744036 & 84,034 & 0,00000000 \\
\hline Log-Lin & 0,91217063 & 0,83205525 & 0,82085894 & 0,03855967 & 74,315 & 0,00000000 \\
\hline Lig-Log & 0,91013715 & 0,82834963 & 0,81690627 & 0,03898275 & 72,387 & 0,00000000 \\
\hline
\end{tabular}

Fuente: elaboración propia 
Tabla 3. Resultado de la estimación del modelo lineal: LIN-LOG

\begin{tabular}{|l|c|c|c|c|}
\hline \multicolumn{1}{|c|}{ Variable } & Coeficientes estimados & $\begin{array}{c}\text { Error } \\
\text { estándar }\end{array}$ & Estadístico t & Valor-p \\
\hline Constante & $-0,74497571$ & 0,15798736 & $-4,71541338$ & 0,00001090 \\
\hline Cooperativismo & 0,00028021 & 0,00142985 & 0,19597335 & 0,84516104 \\
\hline Ingreso & 0,20014244 & 0,01916628 & 10,44242716 & 0,00000000 \\
\hline Educación & 0,00252969 & 0,00447225 & 0,56564153 & 0,57332590 \\
\hline Urbanismo & 0,00259104 & 0,00748670 & 0,34608489 & 0,73024790 \\
\hline Área & $-0,01133192$ & 0,00248676 & $-4,55690409$ & 0,00001977 \\
\hline
\end{tabular}

Estadísticas de la regresión

Coeficiente de correlación múltiple:

Coeficiente de determinación $\mathrm{R}^{2}$ :

$\mathrm{R}^{2}$ ajustado:

Error típico:

Observaciones:
0,92116067

0,84853698

0,83843944

0,02744036

81

Fuente: elaboración propia

Los resultados de la forma LINLIN se presentaron en la tabla 1, y la evaluación del modelo LIN-LOG se presenta en la tabla 3.

Al comparar ambos ajustes (LINLIN y LINLOG), se observa que, a pesar de lo satisfactorio que puedan ser ambos modelos vistos en conjunto (estadístico F), si se analizan las variables cooperativismo, educación y urbanismo individualmente, no son significativas estadísticamente respecto al nivel de confianza del 5\%. Esto es esperable por el conjunto de datos que se utilizaron en el estudio econométrico, pues provienen de una serie de corte transversal y no de una serie de tiempo.

Sin embargo, según establece Gujarati (2011), la baja significancia en estos coeficientes (estadístico t), unida a una alta significancia conjunta (estadístico F), puede deberse a problemas de multicolinealidad o simultaneidad. Para evaluar esta situación puede realizarse una estimación de ambas ecuaciones instrumentalizando dichas variables. No obstante, debe considerarse que la relación entre urbanismo, educación, cooperativismo y desarrollo es compleja, pues no se limita sólo a un impacto lineal, sino que intervienen diversos factores que interaccionan mutuamente.

\section{Análisis de resultados}

Como se observó anteriormente, los ajustes logrados con los modelos son altamente significativos, y en conjunto todas las variables consideradas explican más del $80 \%$ de la variabilidad del IDH cantonal. La multicolinealidad que se puede presentar en el modelo no es un problema cuando se toma el modelo de forma conjunta para predecir el valor del IDH y tampoco invalida los resultados obtenidos en el análisis econométrico, pues, como se indicó, se está trabajando con datos de corte transversal (en un momento en el tiempo) y la significancia de los coeficientes debe evaluarse con mayores niveles de significancia.

Por otra parte, según lo establece Gujarati (2011), la matriz de correlación sintetiza la información necesaria para conocer el grado de asociación entre pares de variables, conocer el grado de independencia condicional y conocer la magnitud de la variabilidad total. Esta matriz de correlación se puede apreciar en la tabla 4. Las correlaciones oscilan desde 0,012 (muy bajas) entre la variable educación y área, y 0,691 entre urbanismo e ingreso. En general, las correlaciones son bajas (menores a 0,5$)$ y llama la atención que la relación entre el nivel de cooperativismo y el ingreso sea negativa, lo cual se repite en el caso de cooperativismo y los niveles de urbanismo y el tamaño del cantón (área). Asimismo, la relación entre el nivel educativo y cooperativismo es directa.

Tabla 4. Matriz de coeficientes de correlación entre variables explicativas

\begin{tabular}{l|ccccc} 
Cooperativas & 1 & & & & \\
Ingreso & $-0,084$ & 1 & & & \\
Educación & 0,327 & $-0,225$ & 1 & & \\
Urbanismo & $-0,048$ & 0,691 & $-0,349$ & 1 & \\
Área & $-0,103$ & $-0,415$ & $-0,012$ & $-0,429$ & 1 \\
\cline { 2 - 6 } & Cooperativas & Ingreso & Educación & Urbanismo & Área
\end{tabular}

Fuente: elaboración propia. 
Estos resultados estarían indicando que el nivel de cooperativismo cantonal es menor conforme aumenta el ingreso de la sociedad, cuanto más urbana sea la sociedad, y cuanto más extenso sea el cantón, aspectos que ameritan una mayor investigación para poder establecer conclusiones.

A partir de la matriz de correlación, es posible conocer no sólo la estructura de asociación entre las variables, sino también la independencia condicional entre pares de variables. Esta independencia se estudia invirtiendo la matriz de correlación, tal como se muestra en la tabla 5.

Tabla 5. Inversa de la matriz de coeficientes de correlación

\begin{tabular}{l|rrrrr} 
Cooperativas & 1,145 & & & & \\
Ingreso & 0,150 & 1,996 & & & \\
Educación & $-0,385$ & $-0,034$ & 1,312 & & \\
Urbanismo & $-0,132$ & $-1,252$ & 0,548 & 2,235 & \\
Área & 0,119 & 0,306 & 0,197 & 0,433 & 1,328 \\
\cline { 2 - 6 } & Cooperativas & Ingreso & Educación & Urbanismo & Área
\end{tabular}

Fuente: elaboración propia

Utilizando la diagonal principal de la inversa de la matriz de correlación, para que cada elemento pueda relacionarse con la proporción de variación de cada variable que puede explicarse por combinaciones lineales de las restantes, es posible determinar que el 12,6\% de la variable cooperativismo puede ser explicado por una regresión sobre las variables restantes (ingreso, educación urbanismo y área). Asimismo, el 49,9\% de la variable ingreso se puede explicar por una regresión de las demás variables.

Lo anterior indica que el nivel de cooperativismo cantonal depende poco de las variables consideradas. No obstante, la variable ingreso se explica en un $50 \%$ por esas variables, incluyendo cooperativismo.

Tabla 6. Correlación parcial entre variables explicativas

\begin{tabular}{l|ccccc} 
Cooperativas & 1 & & & & \\
Ingreso & 0,075 & 1 & & & \\
Educación & $-0,294$ & $-0,026$ & 1 & & \\
Urbanismo & $-0,059$ & $-0,560$ & 0,245 & 1 & 1 \\
Área & 0,090 & 0,230 & 0,149 & 0,326 & 1 \\
\cline { 2 - 6 } & Cooperativas & Ingreso & Educación & Urbanismo & Área
\end{tabular}

Fuente: elaboración propia

Posteriormente, se puede escalar la matriz inversa de correlación hasta obtener valores de unos en su diagonal principal, procedimiento que permite obtener una matriz cuyos elementos fuera de la diagonal corresponden a los coeficientes de correlación parcial entre pares de variables dadas las demás, con el signo cambiado. La tabla 6 presenta los resultados para el modelo LINLOG y muestra que la correlación que parecía existir entre cooperativismo y educación persiste, aunque ha disminuido al tomar en cuenta las otras variables.

De acuerdo con Pulido y Pérez (2001), los modelos semilogarítmicos y logarítmicos representan con mayor fidelidad el proceso de desarrollo o crecimiento de las economías. Por tanto, en la investigación se optó por representar el proceso mediante el modelo LINLOG que permite derivar elasticidades del IDH con respecto a las variables explicativas. No obstante, en este caso los coeficientes de elasticidad afectan la variable explicada por medio del logaritmo de las variables explicativas, de manera que el IDH aumenta más lentamente que los aumentos en las variables independientes (figura 1).

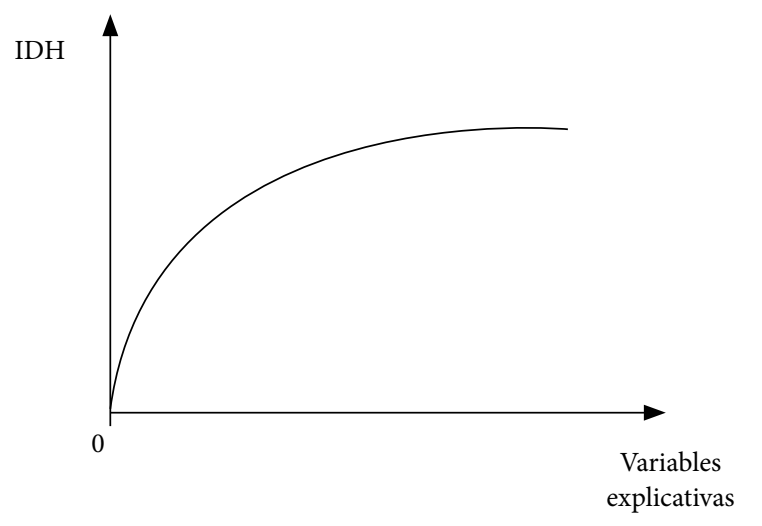

Figura 1. Relación entre el Índice de Desarrollo Humano y las variables explicativas

Fuente: elaboración propia

En la mayoría de los ajustes, las variables de cooperativismo, ingreso, educación y urbanismo (de acuerdo con las dos definiciones analizadas) presentaron signos positivos que denotan su relación directa con el nivel de desarrollo.

La relación del ingreso con el IDH es clara. Un mayor ingreso facilita un mejor acceso a servicios, alimentos y otros insumos de la salud que favorecen el desarrollo. Por otra parte, el factor educacional tuvo un impacto positivo en el desarrollo, debido al mayor y mejor aprovechamiento de los conocimientos e insumos disponibles, que permiten un mayor cuidado de la salud y potencializan las capacidades de las personas.

El signo negativo en la variable extensión se justifica porque entre mayor territorio abarque una comunidad, menor puede ser el acceso de la población a los 
servicios de las empresas cooperativas con que cuenta el distrito principal.

Como se indicó en la evaluación de las formas funcionales, la estructura semilogarítmica (LINLOG) proporciona elasticidades no constantes de la variable explicada con respecto a las variables explicativas. Por esta razón, en la tabla 5 , además de los parámetros estimados con el modelo LINLOG, se incluye el valor de los parámetros obtenidos con la forma funcional logarítmica LOGLOG (ecuación 5).

Lo anterior, por cuanto el ajuste LOGLOG proporciona las elasticidades constantes $\left(\alpha_{\mathrm{i}}^{\prime}\right)$ de la variable explicada con respecto a las variables independientes. En este caso, una alta elasticidad indicaría que un cambio en la variable explicativa tiene un gran impacto sobre el IDH; lo contrario ocurriría en caso de una baja elasticidad (tabla 7).

Tabla 7. Coeficientes estimados para los ajustes del IDH semilogarítmico (LIN-LOG) y logarítmico (LOG-LOG)

\begin{tabular}{|l|c|c|}
\hline \multicolumn{1}{|c|}{ Variable } & LOG-LIN & LOG-LOG \\
\hline Constante & $-0,744976$ & $-2,184005$ \\
\hline Cooperativismo & 0,000280 & 0,000541 \\
\hline Ingreso & 0,200142 & 0,254826 \\
\hline Educación & 0,002530 & 0,004169 \\
\hline Urbanismo & 0,002591 & 0,009040 \\
\hline Área & $-0,011332$ & $-0,014654$ \\
\hline
\end{tabular}

Fuente: elaboración propia

Al observar los coeficientes de elasticidad constantes que proporciona la forma funcional (LOGLOG), se aprecia que el impacto de la variable cooperativismo es el más bajo del set de variables considerado. En este caso es la variable ingreso la que más impacta al desarrollo humano de cada cantón. Por ello, al considerar los impactos más significativos, se deduce que manteniendo las otras variables fijas, un incremento del $1 \%$ en el indicador de ingreso cantonal conduce, en promedio, a un incremento en el IDH del 0,55\%. De igual forma, ceteris paribus, un incremento del $1 \%$ en la población afiliada a las cooperativas conduce, en promedio, a un aumento, de 0,00054\% en el IDH.

Este resultado es importante para el diseño de las políticas de desarrollo regional, dado que muestra la relevancia que posee la inversión pública para levantar los ingresos en cada región.

\section{Conclusiones}

- El modelo propuesto ha permitido enlazar con resultados satisfactorios el proceso de desarrollo (representado por el IDH) con el nivel de la población afiliada a cooperativas, el ingreso cantonal, el nivel educativo y las variables regionales, como el grado de urbanismo y la extensión del territorio.

- El modelo semilogarítmico proporcionó coeficientes de elasticidad "no constantes" que inciden sobre la variable dependiente por medio del logaritmo de la variable independiente, de forma que la variable dependiente aumenta con mayor lentitud que las variables independientes. Este resultado revela que el desarrollo humano cantonal crece a una tasa decreciente con respecto a las variables explicativas consideradas.

- Los modelos indicaron que existe una relación positiva entre el grado de desarrollo regional y el nivel de afiliación de sus pobladores en cooperativas; no obstante, la relación es débil según indicó el estadístico t, el cual no resultó significativo al nivel de 5\%. No obstante, en general dicho estadístico resulta bajo cuando se utilizan datos de corte transversal, por lo cual no se invalida el resultado que muestra la dirección correcta del proceso de desarrollo.

- El modelo en su formulación logarítmica (LOGLOG), permitió derivar los coeficientes de elasticidad constantes de la variable explicada con respecto a las variables independientes. Para el caso específico de la población organizada en cooperativas, el coeficiente de elasticidad resultó bajo.

- Por otra parte, un resultado importante es la correlación entre el nivel de educación y el nivel cooperativo, pues los resultados indicaron que entre mayor es la cobertura de la población con educación secundaria, mayor es el nivel de asociación cooperativa en la región.

- Estos resultados no sólo son relevantes en el diseño de políticas públicas, sino también para las instituciones encargadas de promover el cooperativismo a nivel nacional, pues muestran que la relación empírica entre el nivel de cooperativismo en los diferentes cantones del país incide poco en el nivel de desarrollo humano de dicho cantón.

- El estudio busca evidencia empírica sobre la importancia del desarrollo cooperativo para las regiones del país, siendo de esperar que entre más rico y próspero sea un cantón, mayor será su nivel cooperativo. 
La evidencia recabada apoya esta hipótesis de forma débil, por lo que la hipótesis alternativa deberá ser evaluada: entre más pobre es el cantón, mayor es el nivel de cooperativismo, visto este como un recurso que buscan sus pobladores para superar su nivel de pobreza. Esta suposición cobra interés al considerar que la relación existente entre el nivel de cooperativismo y el ingreso fue negativa, así como entre el cooperativismo y los niveles de urbanismo y el tamaño del cantón (área).

- $\quad \mathrm{Al}$ analizar las correlaciones parciales entre variables, los resultados indican que el nivel de cooperativismo cantonal es menor conforme aumenta el ingreso de la sociedad, entre más urbana es la sociedad y entre más extenso sea el cantón, aspectos que ameritan profundizar en una mayor investigación empírica para poder establecer conclusiones útiles y orientar la política cooperativista.

- Estos son los resultados de una primera aproximación a la búsqueda de evidencia empírica sobre la relación entre desarrollo y cooperativismo. No obstante, el estudio plantea nuevas interrogantes y relaciones por explorar. Es necesario llevar a cabo un estudio detallado del nivel de desarrollo humano y del tipo de cooperativas que poseen los cantones exitosos, así como indagar y analizar el tipo de apoyo que brinda el Estado a estos entes y las razones por las cuales aún no se han convertido en motores de desarrollo humano en sus comunidades.

\section{Referencias}

Aguilar, O. y Fallas, C. (1990). El movimiento cooperativo en Costa Rica. Cenecoop. San José: Imprenta Nacional.

Alianza Cooperativa Internacional (ACI). (2012). Cooperativismo en movimiento. Recuperado de http:// www.centrocultural.coop/blogs/cooperativismo /2012/03/22/2012-ano-internacional-de-las-cooperativas-una-alternativa-para-el-cambio/

Asamblea Legislativa de Costa Rica. (1982). Ley 6756 de Asociaciones Cooperativas y Creación del Instituto de Fomento Cooperativo (Infocoop) de 1982, artículo 2. San José: Imprenta Nacional.

Barro, R. (1991). Economic Growth in a Cross Section of Countries. Quaterly Journal of Economics, 106(2), 407477.

Gujarati, D. (2011). Econometría (4ª ed.). México DF: McGraw-Hill Interamericana de México S.A.

Li Bonilla, F. (1989). Diagnóstico y propuesta de un sistema de información y control gerencial para Fedecredito $R L$ Tesina para optar por el Bachillerato en Cooperativas y Asociativas, Universidad Estatal a Distancia, San José, Costa Rica.

Maddala G. (1996). Introducción a la econometría (2a ed.). México: Prentice-Hall Hispanoamericana S.A.

Programa de las Naciones Unidas para el Desarrollo (PNUD). (2010). Informe sobre desarrollo humano 2009. Madrid: Mundi-Prensa Libros S.A.

Programa de las Naciones Unidas para el Desarrollo (PNUD). Naciones Unidas. A/62/154, Asamblea General. Distr. General. 26 de julio de 2007.

Pulido, A. y Pérez, J. (2001). Modelos econométricos. Madrid: Pirámide. 